Epidemiology

\section{The epidemiology of acute meningitis in children in England and Wales}

\section{K L Davison, M E Ramsay}

\section{A discussion of treatment and prevention options}

A cute meningitis is a disease that causes high levels of anxiety among parents and the medical profession. Meningitis is often caused, however, by organisms that more commonly result in a mild or asymptomatic infection. Young children are most at risk of meningitis, ${ }^{12}$ but the clinical severity also depends on the causative organism; viral meningitis is rarely severe and children tend to make a complete recovery, whereas bacterial meningitis can have a rapid onset, leading to death and serious neurological sequelae. ${ }^{3}$ In the past 50 years in England and Wales, many infections that may cause meningitis have been controlled through routine childhood vaccination programmes. In the short to medium term, the wider use of available vaccines has the potential to prevent even more cases and deaths from this disease. In the longer term, options for further prevention by vaccines against new targets seem promising.

\section{SOURCES OF DATA}

Routine surveillance of meningitis in England and Wales relies on several sources. Clinically diagnosed cases are notified to Consultants in Communicable Disease Control (CCDCs) and passed on to the Public Health Laboratory Service (PHLS) Communicable Disease Surveillance Centre (CDSC). Infections confirmed by isolation from the cerebrospinal fluid (or from a normally sterile site but accompanied by symptoms of meningitis) are also reported by laboratories or referred to the PHLS reference laboratories for further typing. Deaths as a result of meningitis are recorded through the medical certificate of cause of death at the Office of National Statistics (ONS). These routine systems are also used conjointly with enhanced surveillance schemes to monitor meningococcal and pneumococcal meningitis, ${ }^{45}$ and alongside special studies (including the British Paediatric Surveillance Unit "orange card" system (www.bpsu.inopsu.com/)) in the surveillance of neonatal meningitis. ${ }^{6}$ A further source is Hospital Episode Statistics (HES) (www.doh.gov.uk//hes/). These record information on hospital care in NHS hospitals in England.
Diagnoses are recorded at discharge using the World Health Organisation International Classification of Disease codes, currently tenth edition (ICD-10). Cases of meningitis can be identified using the respective ICD-10 codes.

\section{MAIN INFECTIOUS CAUSES OF ACUTE MENINGITIS}

In 2001, 1216 clinically diagnosed cases of meningitis in children in England were formally notified to the PHLS CDSC. Meningococcal meningitis was the most common reported cause $(48 \%)$, with other bacteria (12\%), viruses (19\%), ing for the rest. These proportions are unlikely to reflect true differences in incidence, however, since the completeness of notification is better for more serious diseases. For example, notifications are relatively complete for meningococcal meningitis, ${ }^{7}$ probably because of the immediate need to confirm and report the case for public health action. In contrast, viral meningitis is probably under-diagnosed and under-notified. HES records around 600 admissions for viral meningitis in children in England each year, which suggests that notifications under-estimate the burden of meningitis requiring admission to hospital by approximately 160\%. Data from laboratories are also prone to bias because of different methods of confirmation and different clinical practices. Viral meningitis is likely to be relatively under-estimated as, with the exception of herpes simplex virus (HSV), ${ }^{8}$ the specific aetiology is unlikely to alter the management of the case in view of the limited treatment options readily available.

Mycobacterium tuberculosis can cause meningitis, but does not generally present in the acute phase and is therefore not included here.

\section{VIRAL MENINGITIS}

Many viruses are known to cause meningitis in children. Before the introduction of the combined measles, mumps, and rubella vaccine (MMR) in 1988, mumps was the commonest cause of childhood meningitis in England and Wales. Epidemics occurred every three years, and in and other or unknown causes account-
1987 general practitioner consultations reported through the Weekly Returns Service of the Royal College of General Practitioners estimated an overall incidence of approximately 21 per 100000 (all ages). By 1997 this had fallen to less than 1 per $100000 .^{9}$ Estimates of the number of admissions to hospital for mumps, usually for viral meningitis, averaged around 1200 per year in the pre-vaccine era ${ }^{10}$; more recent data suggest that fewer than 100 cases per year occur (www.doh.gov.uk//hes/). Reports of mumps virus isolates from the CSF also declined dramatically, indicating a fall in the number of cases of confirmed mumps meningitis. In the post MMR vaccine era, enteroviruses are thought to be the most common group of viruses to cause meningitis in England. ${ }^{91-15}$ Less common causes include herpes viruses, lymphocytic choriomeningitis (LCM), cytomegalovirus (CMV), the adenoviruses, arboviruses, influenza, and rubella. ${ }^{11}$ Deaths from viral meningitis are rare. ${ }^{16}$

\section{Enterovirus meningitis}

There are around 70 different types of enteroviruses, but those most likely to cause meningitis are polio, coxsackie (types A and B), and ECHO (enteric cytopathogenic human orphan) viruses, ${ }^{17}$ although there is no longer any indigenous polio in Europe. Transmission is primarily via the faecal, oral, and respiratory routes. Virus is shed in stools and can be detected for weeks after infection. The virus inhabits the intestinal tract, causing gastrointestinal diseases, but once in the bloodstream it can show affinities for many organs, including the brain. ${ }^{17}$ Most confirmed infections reported are isolates from faecal specimens and are not associated with infection of the central nervous system. Weekly totals of laboratory reports of enterovirus isolates, however, parallel clinically notified cases of viral meningitis (fig 1), with summer peaks occurring when particularly virulent strains are circulating. ${ }^{12} 18$ This supports the hypothesis that enteroviruses are the main cause of notified viral meningitis. There is currently no recommended treatment for enteroviral infections widely available, although a new treatment, pleconaril, has shown some promising results under trial. ${ }^{2}$ Preventative methods such as good hygiene and sanitation are recommended.

\section{BACTERIAL MENINGITIS}

The most common causes of bacterial meningitis in children in England and Wales are meningococcal, Haemophilus influenzae type b (Hib), and pneumococcal infections. Less common causes include group B streptococci, Escherichia coli, Listeria sp., and staphylococcus. 


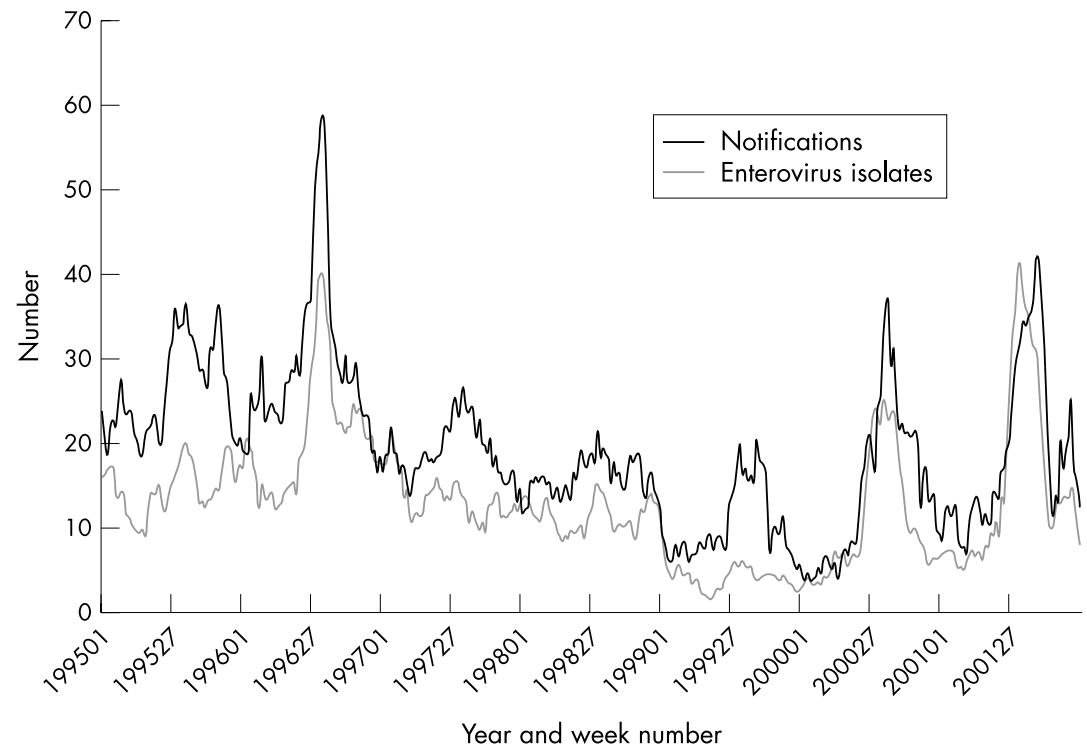

Figure 1 Notifications of viral meningitis and reports of enterovirus isolates to the Public Health Laboratory Service; five week moving average, England and Wales, 1995-2001.

Between 1982 and 2001, the most common bacterial isolate from the CSF reported to the PHLS was Neisseria meningitidis (fig 2).

\section{Hib meningitis}

Hib was once the leading cause of bacterial meningitis in children, with most cases in children under 5 years of age. ${ }^{19}$ Vaccination has virtually eradicated Hib meningitis in England and Wales. Since routine infant immunisation began in 1992, few isolates of Haemophilus influenzae from the CSF have been reportedmany of these caused by non-capsulated organisms (fig 2). Following the success of conjugate vaccines against Hib, similar vaccines have now been developed against serogroup $\mathrm{C}$ meningococcal infection and against the major serotypes of $S$ pneumoniae.

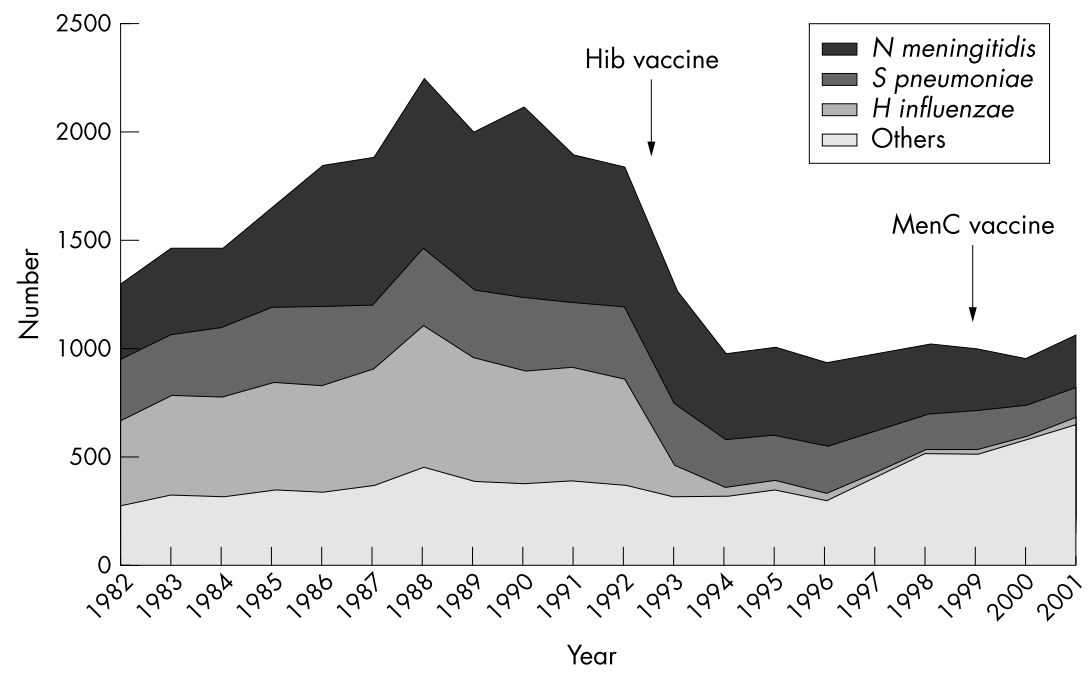

Figure 2 Reports of bacterial isolates from the cerebrospinal fluid to the Public Health Laboratory Service in England and Wales, 1982-2001. and septicaemia. This came against a background change in clinical practice away from performing lumbar punctures ${ }^{21}$ which meant CSF isolates of $N$ meningitidis started to decline (fig 2). The trend in CSF isolates was in contrast to increases in the overall number of confirmed cases (http://cdsc.hpa.org.uk/ topics az/meningo/data meni-t3a.htm).

In November 1999, the UK became the first country to introduce the meningococcal serogroup $\mathrm{C}$ conjugate vaccine (MenC) into their national childhood immunisation programme and at the same time started to offer vaccine to all children under 18 years. Eighteen months after the campaign began the number of laboratory reports of serogroup C fell by over $80 \%$ in the targeted groups. ${ }^{22}$ Meanwhile, cases of serogroup B have remained high (http:// cdsc.hpa.org.uk/topics_az/meningo/data meni-t3a.htm).

\section{Pneumococcal meningitis}

Pneumococcal meningitis tends to occur in children less than 1 year. ${ }^{23}$ The overall case fatality ratio for pneumococcal meningitis is over $20 \%$, and may be even higher in very young children. ${ }^{23}$ Therefore, although pneumococcal meningitis is less common than meningococcal meningitis, it is a leading cause of certified death as a result of bacterial meningitis (source: Office of National Statistics (ONS)). In 1993, deaths in children as a result of pneumococcal meningitis represented $25 \%$ of all bacterial meningitis deaths certified to ONS-by 2000, this proportion had increased to $38 \%$. Also, high rates of sequelae following pneumococcal meningitis have been documented. $^{24}$ Conjugate vaccines against the main serotypes of pneumococcal meningitis are currently offered only to high risk children. ${ }^{25}$

\section{DISCUSSION}

Acute meningitis in children in England and Wales is caused by a variety of organisms. The severity of disease is generally milder for viral than bacterial infections, but depends on other factors such as age, disease presentation, and causative organism. Although antibiotic treatment has had a significant impact on deaths from bacterial meningitis, treatments for viral infections have been limited. The continued effectiveness of antibiotics is a potential problem because of the emergence of resistance, particularly for pneumococcal meningitis. ${ }^{23}$ Vaccination has had a significant impact on the prevention of mumps, and Hib and serogroup C meningococcal meningitis, which are now at very low levels; however, if MMR coverage remains at its current level of $84 \%,{ }^{26}$ or declines further, the re-emergence of mumps meningitis is possible. The current main causes of morbidity and mortality from meningitis in 
children in England and Wales are serogroup B meningococcal and pneumococcal infections. The wider use of conjugate vaccines against pneumococcal infection, has the potential to reduce the number of cases of invasive pneumococcal diseaseincluding meningitis - within a few years of implementation. Efforts to produce a safe and effective vaccine against a broad range of serogroup $B$ meningococcal strains have now produced several possible candidates with the potential aim of the virtual elimination of meningococcal meningitis. ${ }^{27}$

\section{Arch Dis Child 2003;88:662-664}

\section{Authors' affiliations}

K L Davison, M E Ramsay, Public Health Laboratory Service Communicable Disease Surveillance Centre, London, UK

Correspondence to: $\operatorname{Dr}$ M E Ramsay, Consultan Epidemiologist, Immunisation Division, Public Health Laboratory Service Communicable Disease Surveillance Centre, 61 Colindale Avenue, London NW9 5EQ, UK; Mramsay@phls.org.uk

\section{REFERENCES}

1 Noah ND. Epidemiology of bacteria meningitis: UK and USA. In: Bacterial meningitis. London: Academic Press, 1987:93-115.

2 Rotbart HA. Viral meningitis. Semin Neurol 2000;20:277-92.

3 Fellick JM, Thomson AP. Long-term outcomes of childhood meningitis. Hosp Med 2002;63:274-7.
4 CDSC. Enhanced surveillance of suspected meningococcal disease. Commun Dis Rep CDR Wkly 1999;9:78-9

5 George RC, Melegaro A. Invasive pneumococcal infection: England and Wales, 1999. Commun Dis Rep CDR Wkly [serial online] 2001

6 Anon. Neonatal meningitis in England and Wales: 10 years on. Arch Dis Child Fetal Neonatal Ed 2002;84:F85-9.

7 Davison KL, Ramsay ME, Crowcroft NS, et al. Estimating the burden of serogroup $C$ meningococcal disease in England and Wales. Commun Dis Public Health 2002;5:213-19.

8 Kesson AM. Use of aciclovir in herpes simplex virus infections. J Paediatr Child Health 1998;34:9-13

9 Fleming DM. Weekly returns service of the Royal College of General Practitioners. Commun Dis Public Health 1999;2:96-100.

10 Galbraith NS, Young SE, Pusey JJ, et al. Mumps surveillance in England and Wales 1962-81. Lancet 1984;1:91-4.

11 Connolly KJ, Hammer SM. The acute aseptic meningitis syndrome. Infect Dis Clin North Am 1990;4:599-622.

12 Maguire HC, Atkinson $P$, Sharland $M$, et al. Enterovirus infections in England and Wales: laboratory surveillance data: 1975 to 1994. Commun Dis Public Health 1999;2:122-5.

13 Wang VJ, Kuppermann N, Malley R, et al. Meningococcal disease among children who live in a large metropolitan area, 1981-1996. Clin Infect Dis 2001;32:1004-9.

14 Stanwell-Smith RE, Stuart JM, Hughes AO, et al. Smoking, the environment and meningococcal disease: a case control study. Epidemiol Infect 1994;1 12:315-28.

15 Kolski H, Ford-Jones EL, Richardson S, et al. Etiology of acute childhood encephalitis at The Hospital for Sick Children, Toronto,

1994-1995. Clin Infect Dis 1998;26:398-409.

16 Dedicoat M, Muir D. Viral meningitis-or encephalitis. Practioner 2000;242:489-92.
17 Brown EH. Enterovirus infections. BM 1973;2:169-71.

18 CDSC. Viral meningitis associated with increase in echovirus type 13. Commun Dis Rep CDR Wkly 2000;10:277,280.

19 Nazareth B, Slack MP, Howard A, et al. A survey of invasive Haemophilus influenzae infections. Commun Dis Rep CDR Rev 1992;2:R13-16.

20 Kaczmarski EB, Ragunathan PL, Marsh J, et al. Creating a national service for the diagnosis of meningococcal disease by polymerase chain reaction. Commun Dis Public Health 1998;1:54-6.

21 Ramsay M, Kaczmarski E, Rush M, et al. Changing patterns of case ascertainment and trends in meningococcal disease in England and Wales. Commun Dis Rep CDR Rev 1997; 7:R49-54.

22 Miller E, Salisbury D, Ramsay M. Planning, registration, and implementation of an immunisation campaign against meningococcal serogroup $C$ disease in the UK: a success story. Vaccine $2001 ; 20$ (suppl 1): $558-67$

23 Laurichesse $\mathbf{H}$, Grimaud $O$, Waight $P$, et al Pneumococcal bacteraemia and meningitis in England and Wales, 1993 to 1995. Commun Dis Public Health 1998; 1:22-7.

24 Pikis A, Kavaliotis J, Tsikoulas J, et al. Long-term sequelae of pneumococcal meningitis in children. Clin Pediatr (Phila) 1996;35:72-8

25 Chief Medical Officer. Extending meningitis vaccine to 20-24 year olds; pneumococcal vaccine for at risk under 2 year olds. http://www.doh.gov.uk/cmo/cmo0201.htm 2002.

26 CDSC. COVER Programme: July to September 2002. Commun Dis Rep CDR Wkly [serial online] 2002.

27 Jodar L, Feavers IM, Salisbury D, et al. Development of vaccines against meningococcal disease. Lancet 2002;359: 1499-508. 